Drs. Spengler and Unger, who have kindly furnished me with a report; of which, however, I can only give a very short summary. Of these 35 cases, only I, belonging to a consumptive family, offered no distinct signs to auscultation and percussion, although the spirometer shewed a considerable impairment of the respiratory capacity. Of the other 34 cases, 19 were in the first stage, with distinct deposits; 15 in the second, with cavities. Of the 19 in the first stage, 2 had deposits in both sides, and some had other complications, as pleuritic effusion; 7 of the 19 left cured, 6 improved, 6 remained under treatment.; 18 of the 19 had gained in weight; 8 had previously had attacks of hæmoptysis, which only in one of them returned in a very slight degree during the stay. Attacks of fresh colds were remarkably rare in all of them. Of the 15 in the second stage, only 2 were free from fever; 3 of them had pleuritic exudations; 5 of the 15 died ( 3 from gradual consumption, 2 from hæmoptysis); I left worse than he arrived; I improved; 2, who had arrived with considerable pulmonary distension, are steadily im. proving; and $I$ is regarded as perfectly convalescent. It is worthy of remark, that diarrhœea did not occur in any of them, and that the night-perspiration in almost all cases disappeared after a short stay. In one case only, careless exposure led to a croupous pneumonia of the left lower lobe, with a favourable termination on the fifth day.

The treatment adopted at the Davos consists in the use of much milk, and light nourishing food; a moderate amount of wine, principally the red wine of the Valteline; and graduated exercise, first on level, later up hill. The cold douche is likewise in many cases used with advantage.

Those who consider the facts related will probably not deny that they offer testimony in favour of mountainous climates. The two cases which at a later period ended fatally are particularly instructive, by shewing that a curative process had taken place during the stay in elevated regions. Both cases, and probably also the three others related, belonged to the class described by Addison under the head of pneumonic phthisis. It is probably on these low forms of catarrhal pneumonia, with a tendency to chronic infiltration, cheesy transformation, and formation of cavities, that the Alpine climates have a truly curative and prophylactic influence.

The question, how the mountain climates exercise so favourable an influence, can probably not be answered without entering the field of theories, which I wish to avoid as much as possible. There are, however, some undeniable facts. Thus the air of elevated regions is lighter, more rarefied, and cooler; and it is usually free from the foreign admixtures found in towns, and also free from the various kinds of malaria. The food, the occupation, and the whole manner of living, are generally different from those prevailing in large towns and much inhabitci : istricts. The influence observed by most people on their removal to mountainous climates is an improvement of appetite, digestion, formation of blood, and nutrition in general. Exhilaration and increased inclination to exercise are likewise frequent attendants of the change. All the influences enumerated, to which others might be added, are of importance in the consideration of the question before us. I will only allude to one; viz., the rarefied air. A given volume of air contains absolutely less oxygen and other constituents at an elevation of 5,000 feet, than at the level of the sea. The movements of the thorax must, therefore, be increased, if the same amount of oxygen is to be introduced; or a smaller amount of oxygen will be introduced, if the movements of the thorax remain the same. It has been asserted, but, as far as I know, not yet been proved experimentally, that the inspirations on mountains are deeper. Healthy persons, at all events, usually have an inclination to take deeper inspirations; and the broad chest of mountaineers may be regarded as an effect of increased respiratory movements. If this view were correct, it might be asked, how far this augmented action of the organs of respiration, and, as a necessary consequence, those of circulation, influences the sanguifcation and all the processes of nutrition-how far, I say, this mere increase of chest-expansion would go to explain the facts before us. All influences, climatic, physical, or mental, by which the breathing is habitually increased, appear to act beneficially in the tendency to consumption; while all those causing diminished breathing seem to favour, in man and animals, the development of scrofulous and catarrhal inflammations, and of consumptive diseases of the lungs in general.

Although I have already occupied too much space, I must still add a word of caution. Not all consumptive constitutions probably are fit for the Alpine climate, and not all the stages of consumptive disease may be benefited. The invalid ought not to be allowed to go to any Alpine climate he likes, and do there as he pleases; but he ought to be directed to a place where he can have the advice of a medical man who will carefully superintend his diet, his exercise, and his whole manner of living. Many invalids lose the benefit obtained in the commencement by over-exercise and careless exposure; and the very help which these climates have given them may be turned by them to their ruin. 'It is further necessary that the invalid be sent in an early stage of the disease, and that he remain long enough, if possible, to regain the full capacity of healthy lungs; and that he be not allowed to return too early to his former unhealthy residence and occupation. On all these points, however, I cannot suggest better advice than that embodied in the excellent works of Sir James Clark on the Sanative Influence of Climate, and on Pulmonary Consumption.

\section{A CASE OF INVERSION OF THE UTERUS.}

\section{By G. F. HODGSON, Esı., Brighton.}

A TRADEsMan's wife, a delicate woman, aged 27 , in confinement with her second child (the first having been born two years previously, with the aid of forceps, and then dead), sent for me on the 5th January, 1867. She had then been in labour about four hours, the pains being of average frequency and moderate in severity. The child's head was still above the brim; but the os uteri was so low down and so very patent and flaccid (as was also the os externum) that I thought it right to stay with the patient. Three hours, however, afterwards elapsing without any advance being made, I then absented myself to visit a few urgent cases, first leaving word where I might be found. On returning in about an hour, I was met in the street by the husband, who reported the child as already born. On my arrival in the room, I found the child separated by the nurse and on the hearth-rug, and the nurse herself at the bedside, holding on by the cord, which she declined to let go of, until $\mathbf{I}$ should take it of her; as if she thought that it would slip up and be lost, if tight hold were not kept of it. Observing a large amount of blood in the bed, I hastily passed my hand over the abdomen to ascertain the absence of a twin (but did not examine for the uterus more precisely), gave the patient a close of ergot, and directed a friend of hers to press her hypogastrium whilst I proceeded to remove the placenta. This I found already presenting at the os externum, and on my making gentle traction by the cord, it readily passed into my hands; not, however, in the usual flaccid form, but in a firm, solicl, and apparently much larger mass, some of which still remained in the vagina, and was ascertained to be of smaller and smaller bulk the higher the finger proceeded to trace it. This led. me to suspect that the uterus was inverted, and to search for the fundus uteri in the hypogastrium ; and failing to find it there, I was convinced of the nature of the case. At this moment, the patient's friend whispered to me that she seemed to be dying; and certainly she was in a state of utter collapse. I therefore dispatched the husband to the Old Steine fo: a second medical man, and he soon returned with Dr. Hall.

In the meantime I arranged for the patient to have a liberal supply of brandy egg-flip and other cordials, and then again gave my attention to the placenta. It was extensively adherent to, and therefore enveloping, the inverted fundus uteri, and had to be peeled from it. When I had accomplished its removal, I returned the uterus into the vagina, and then endeavoured to re-invert it within itself, at which I had made bat little progress when Dr. Hall entered the room. At this stage the hæemorrhage was slight, but whether this was owing to the syncope, or to the special condition of the uterus itself, I cannot tell. Dr. Hall quickly examined the case, and promptly agreeing with me as to its nature (the fundus uteri still being undetectable in the hypogastrium) he took a turn at the taxis, and in a few minutes-with his fingersaccomplished it most satisfactorily. The vagina and os uteri then appeared so intenseiy relaxed as to make us apprehend that a relapse would occur, wherefore we plugged the vagina with a good-sized sponge, previously wrung out of cold vinegar and water, and secured it by a perineal band attached to the abdominal binder. The collapse of the patient continued so extreme that I was obliged to remain with her for several hours, giving stimulants and small doses of opium with quinine at short intervals. She passed a better night than might have been expected, and the next day had fairly rallied. The sponge was then carefully removed, and found to be but slightly soaked with hæemorrhage. The lochia afterwards proved scanty, but fotid, requiring disinfecting ablutions. The patient re-acquired her strength but very gradually; and, although she did not attempt to suckle the child, only left her room for the first time at the end of a month, and continued the use of tonics and extra diet for some time subsequently. Menstruation re-occurred two months after the parturition, and has been "regular" ever since, the patient being now (six months since her confinement) in other respect: also quite well for her.

I think there can be no doubt that the inversion of the womb was, in this case, caused by the nurse pulling violently at the funis (the placenta being adherent to the fundus), and that it was favoured by the very flaccid and patent condition of the os uteri, which I detected at 
my first visit, and by the sudden deiivery that happened, as the nurse and her friend both declared that the patient had only one bad pain, and that therefore there was not time to send for me.

The Edinburgh Medical Fouriul for May 1867, contains a rather voluminous paper on "the production of inverted uterus", by Dr. Matthews Duncan, which he sums up by describing four kinds, viz. -

I. Spontanious Passize Iniersion, which occurs in cases of inertia of the uterus, the organ being large, lax, and capable of being inverted by little force, that force being the patient "bearing down" strongly.

2. Artificial P'assiz' Int'irsion, which occurs under the same circumstances as the first kind, only, instead of the force being the patient "bearing down", it is exercised by the attendant, either above by pushing, or below by pulling at the funis or placenta.

3. Spontunious Actic' Inz'crsion (which modern authorship treats of as the most common kind, but therein, Dr. Inuncan is of opinion, overstates the truth), occurs from irregular action of the uterus. The fundus being relaxed, it is projected by "bearing down", or by the retentive power of the abdomen, into the uterine cavity, and the lateral walls contracting upon it cause it to descend more and more until it is expelled through the os.

4. Artificial Actia'e Ina'rsion, which occurs under the same condition of the uterus as the third kind, but the force applied is the same as in the sccond kind.

Dr. Duncan says, that in the two first kincls, hemorrhage will occur, if the placenta be separated.

In the same Journal for July 1867 , is contained a case of inversion of the utcrus, as read before the Edinburgh Obstetrical Society by Dr. Inglis, which lead to an interesting discussion, during which Sir J. Simpson made the remarkable statement that he had never seen a case of inverted uterus occur, but only had been called to them days, weeks, or months afterwards.

I may add, that the case which I have narrated above, was the first of its kind that either Dr. Hall or myself had met with.

\section{PROGRESS OF MEDICAL SCIENCE.}

\section{MIDWIFERY AND DISEASES OF WOMEN.}

Removal of the Uterts and its Appexdages in Procidentia CTERI. Dr. S. Choppin relates (Southern Fourn. Mid. Sciences, Feb. I867) a case of this. The subject of it was a woman, 38 years of age. The tumour was six inches in length and three and $a$ half in breadth. The operation for narrowing the vagina having failed to afford relief, Dr. Choppin determined to remove the uterus and its appendages. Accordingly, on January 12 th, $186 \mathrm{I}$, the patient being brought under the influence of chloroform, Dr. Choppin operated in the following manner. The tumour was seized by a pair of vulsellum-forceps, implanted in the neck of the uterus, dragged down as far as possible, and held steadily by an assistant. A circular incision was then made through that portion of the vagina attached to the neck of the uterus, so as to conpletely separate it, with a view of drawing the organ down and separating it from its peritoneal attachment; but he was thwarted in his attempt, by adhesions which were found to exist anteriorly between the anterior wall of the vagina and uterus to the posterior wall of the bladder, and posteriorly to the lower wall of the vagina and anterior walls of the rectum. By a careful dissection he severed the anterior and posterior adhesions, thus permitting further traction downward of the uterus, and exposing a pedicle made up of the peritoneal attachments of the organ. The hxmorrhage, which thus far had been quite profuse, was arrested before proceeding any further. The loop of Chaissaignac's cicraseur was now thrown around the peritoneal attachments and gradually tightened, during a period of twenty-five minutes, when its division was completed, and the uterus, left Fallopian tube, and left ovary removed. It was found that the right ovary had not descended, and, consequently, the right Fallopian tube was comprised in the pedicle of the tumour, and severed near the body of the uterus. Their removal not being considerel necessary to the success of the operation, they were allowed to remain in the abdomen. No blood followed the use of the ecraseur. At this stage of the operation, hernia of a loop of intestine took place. 'This was reduced, and further descent prevented by pressure of the hand of the assistant over the vulva, until permanent closure of the opening in the vagina could be effected. This was done by means of sims' clamp-suture. The inverted vagina was then reduced, the patient removed to her bed, and placed upon her back with the pelvis elevated, and a full dose of opium administered. The patient rested well all that night. The next day no febrile reaction whatever occurred, although she complained of some tenderness or soreness over the abdomen. On the third day, suppuration began, but an examination by the speculum showed that most of the vaginal wound had healed by first intention. The suppuration, however, continued, quite profuse at times, for about three weeks, when the wiresutures and clamps came away. She was again examined with the speculum, and complete cicatrisation of the wound was found to have taken place. The general health of the patient began rapidly to im. prove from that moment. On February 19th, she was presented, in Dr. Choppin's clinical lecture, to the class of the New Orleans School of Medicine, thus demonstrating that the uterus could be removed without causing death. The patient remained under observation until the following April, during which time her condition improved, so that she presented, at the time of leaving the infirmary, a robust and healthy appearance. Returning to labour and usefulness, she continued to enjoy good health until the spring of 1864 , when she succumbed to an attack of dysentery.

\section{ANATOMY, PHYSIOLOGY, AND PATHOLOGY.}

Absorption from Batus.-M. Hoffmann has been conducting a series of experiments with mineral salts and other organic matters, which leads him to the following conclusions. 1. Chemical and other agents dissolved in water penetrate slowly but surely into the animal economy by the skin; and it is only when the blood and other fluids are saturated by them that the organism expels them. 2. All medicamental agents are not absorbed to the same extent by the skin. 3. The contradictory results hitherto obtained, are the effect of the insufficient duration of the experiments. - Repertoir' de Pharmacic.

Structure and Functions of Gland-Cells.-Professor J. H. Salisbury of Cleveland, Ohio, communicates to the American Fournal of Medical Sciences observations on rheumatism in connexion with the structure and function of parent gland-cells. He says that each individual parent gland-cell has all the elements of an independent organism, possessing a vitality independent of that of the systemic life. Each cell feeds, digests its food, assimilates it, grows, organises, and eliminates. In its normal condition it organises those cell, and filamentous, and other products that are needed for the physiological tissues. When its food is imperfect, its processes of digestion, assimilation, and organisation become deranged, and pathological products are the result. These pathological products vary with the varying conditions. He distinguishes four or more types of rheumatism: viz., I. Lithic rheumatism. 2. Oxalic rheumatism. 3. Cystine rheumatism. 4. Phosphatic rheuma tism. The causes which, under certain conditions, produce the various
forms of rheumatism, under other circumstances result in a variety of mental, paralytic, and neuralgic disturbances.

Action of Muscular Tissue as observed by the Myograph. - The results obtained by the use of Marey's myograph have been called in question by $\mathbf{M}$. Rouget, a very distinguished French histologist. M. Rouget, who has been examining muscular contractions with the assistance of the microscope, does not corroborate the opinion lately expressed that, during contraction, the muscular fibre is in a state of vibration. On the contrary, he states that permanent contraction does not consist of a series of successive shocks or waves. The muscles of living animals in a state of sustained contraction, appear perfectly motionless when examined by the microscope. The undulations traced by the myograph exist, according to $M$. Rouget, only during the period of variable contraction when the exciting influence has not displayed suffi. cient intensity to call the muscle into complete contraction. When, for example, a powerful electric current is substituted for a weak one, the vibrations, before evident, entirely disappear, the muscle remaining in a perfectly rigid state. - London Revieio.

New Rfsearches on tile Cardic Circulation of Animals. Dr. Judée has just published a pamphlet on this subject. He shows that in frogs what is, by common consent, called first movement, is compounded of the contraction of the auricular portion and the dilatation of the ventricle; that the second is formed by the contraction of the ventricle and the dilatation, per contra, of the two auricles with which the heart of this batrachian is provided. In the second part of the book, relying not only on his own experiments on frogs, but on those made on horses by MM. Chauveau and Marey, M. Judée stated that what these physiologists have taken for the commencement of the first move. ment, or systole, was nothing but the end of the second, or diastole, of the heart. In other words, that the systole of the auricle does not form part of the systole of the ventricle, but of its diastole ; so that, in fact, in the horse, at least, the cardiac revolution does not commence, as is 